\title{
A Matriz SWOT e suas Subdimensões: Uma Proposta de Inovação Conceitual
}

\author{
The SWOT Matrix and its Subdimensions: A Conceptual Innovation Proposal \\ La Matriz SWOT y sus Subdimensiones: Una propuesta de innovación conceptual
}

Recebido: 02/02/2021 | Revisado: 06/02/2021 | Aceito: 06/02/2021 | Publicado: 14/02/2021

\author{
João Florêncio da Costa Júnior \\ ORCID: https://orcid.org/0000-0002-3962-1010 \\ Universidade Potiguar, Brasil \\ E-mail: jfcj1977@gmail.com \\ Diogo de Menezes Cortês Bezerra \\ ORCID: https://orcid.org/0000-0002-8719-106X \\ Universidade Potiguar, Brasil \\ E-mail: diogo.bezerra@unp.br \\ Eric Lucas dos Santos Cabral \\ ORCID: https://orcid.org/0000-0003-2855-7049 \\ Universidade Federal do Rio Grande do Norte, Brasil \\ E-mail: ericlucascabra194@gmail.com \\ Rosângela Carmelita Pessoa Moreno \\ ORCID: https://orcid.org/0000-0001-5074-4057 \\ Universidade Potiguar, Brasil \\ E-mail: rosangela.moreno@unp.br \\ Ahanna Kiaara Souza Pires \\ ORCID: https://orcid.org/0000-0002-8854-2255 \\ Universidade Potiguar, Brasil \\ E-mail: ahannakiaara@gmail.com
}

\begin{abstract}
Resumo
O presente trabalho objetiva propor uma releitura da análise SWOT, apresentando um modelo adequado às demandas e transformações das organizações modernas, focando em uma definição mais robusta e específica das categorias da matriz SWOT - forças fraquezas, oportunidades e ameaças. A pesquisa foi dividida em duas etapas: i) pesquisa bibliográfica e documental; e ii) pesquisa-ação com observação participante. Durante o período de 2014 a 2016 em uma empresa Britânica no ramo de Business Intelligence, pôde-se avaliar o progresso do uso e da adaptação da análise SWOT às demandas de competitividade. Baseados nas evidências documentais e empíricas, os autores propuseram uma releitura da MATRIZ SWOT em que cada uma das dimensões originais é subdividida em cinco subcategorias, oferecendo assim, uma ferramenta para dar suporte a análises mais detalhadas e robustas. As forças e fraquezas são divididas nas seguintes categorias: i) sintomáticas; ii) adormecidas; iii) constantes; iv) circunstanciais; e iv) tangíveis. As oportunidades são divididas nas seguintes categorias: i) exploradas; ii) passageiras; iii) desperdiçadas; iv) inacessíveis; e v) disfarçadas. Por fim, as ameaças apresentam as seguintes subcategorias: i) constantes; ii) passageiras; iii) adormecidas; iv) provocadas; e v) disfarçadas. Este trabalho contribui com a literatura e a prática da gestão estratégica ao oferecer uma alternativa para endereçar as dificuldades de precisão na definição das categorias da Matriz SWOT, criando subcategorias até então não levantadas na literatura para que se possa mais precisamente abarcar forças e fraquezas e melhor avaliar oportunidades e ameaças, validando assim, a continuidade do uso desta ferramenta.
\end{abstract}

Palavras-chave: Matriz SWOT; Tomada de decisão; Estratégia; Ferramentas estratégicas.

\begin{abstract}
The present work endeavours to propose a reinterpretation of the SWOT Matrix, through a new model adapted to modern organizations' demands and renovations, focusing on a more robust and specific definition of its categories strengths, weaknesses, opportunities and threats. The research was divided into two stages: i) bibliographic and documentary research; and ii) action research with participant observation. It took place in a British company specialised in business intelligence between the years of 2014 to 2016, wherein the progress of the utilization and adaptation of the SWOT analysis to the competitive demands of the organization was evaluated. Based on documentary and empirical evidence, the authors proposed a re-reading of the SWOT Matrix in which each category is divided into five subcategories, offering guidance to support a more robust analysis. The strengths and weaknesses are divided into the following categories: i) symptomatic; ii) asleep; iii) constant; iv) circumstantial; and iv) tangible. Opportunities are divided into the following categories: i) explored; ii) temporary; iii) wasted; iv) inaccessible; and v) disguised. Finally, threats were classified as i) constant; ii) temporary; iii) asleep; iv) provoked; and v) disguised. The article contributes to the literature and practice of strategic management by offering an alternative to address the difficulties in defining the categories within the SWOT Matrix, creating subcategories not previously raised in the
\end{abstract}


literature so that it can embrace with more precision strengths and weaknesses and better evaluate opportunities and threats, thus validating the continued use of this tool.

Keywords: SWOT matrix; Decision-making; Strategy; Strategic tools.

\section{Resumen}

El presente trabajo tiene como objetivo proponer una relectura del análisis SWOT, presentando un modelo adecuado a las demandas y transformaciones de las organizaciones modernas, enfocándose en una definición más robusta y específica de las categorías de la matriz SWOT - fortalezas, oportunidades, debilidades, amenazas. La investigación se dividió en dos etapas: i) investigación bibliográfica y documental; e ii) investigación-acción con observación participante. Durante el período de 2014 a 2016 en una empresa británica del ramo de Business Intelligence, se pudo evaluar el progreso del uso y adaptación del análisis SWOT a las exigencias de competitividad. Con base en evidencia documental y empírica, los autores propusieron una relectura de la SWOT en la que cada una de las dimensiones originales se subdivide en cinco subcategorías, ofreciendo así una herramienta para sustentar análisis más detallados y robustos. Las fortalezas y debilidades se dividen en las siguientes categorías: i) sintomáticas; ii) dormido; iii) constantes; iv) circunstancial; iv) tangible. Las oportunidades se dividen en: i) exploradas; ii) pasajeros; iii) desperdiciado; iv) inaccesible; v) disfrazado. Finalmente, las amenazas se dividen en: i) constantes; ii) pasajeros; iii) dormido; iv) provocado; v) disfrazado. Este trabajo contribuye a la literatura y la práctica de la gestión estratégica al ofrecer una alternativa para abordar las dificultades de precisión en la definición de las categorías de la Matriz SWOT, creando subcategorías hasta ahora no planteadas en la literatura, validando así el uso continuado de esta herramienta.

Palabras clave: Matriz DAFO; Toma de decisiones; Estrategia; Herramientas estratégicas.

\section{Introdução}

A análise SWOT que explora os ambientes internos e externos das organizações acerca de seus pontos fortes e fracos, oportunidades e ameaças - é uma das ferramentas estratégicas mais utilizadas em todo mundo (Eastwood et al, 2016; Ghazinoory; Abdi; Azadegan-Mehr, 2011; Helms; Nixon; 2010). Seu uso intensivo se dá devido à facilidade com que pode ser empregada e à sua natureza funcional e intuitiva; porém, tal facilidade traz em si severas limitações, tais como o subjetivismo, a ausência clara e explicita de uma hierarquia de priorização e, principalmente, a imprecisão das definições dos fatores ou dimensões: forças, fraquezas, oportunidades e ameaças (Lewa; Narikae, 2017; Ghazinoory; Abdi, and Azadegan-Mehr, 2011; Helms and Nixon, 2010; Middleton, 2003, Pickton and Wright 1998).

Dada sua natureza aglutinatória, a análise SWOT, pode ser usada nas mais diversas conjunturas em diferentes organizações enquadrando os elementos-chave em vários campos de atividade e diferentes níveis de interesse tais como liderança, estratégia, processo de produção, marketing externo e interno, valor da marca, desenvolvimento de produto, distribuição, modelo de negócios, e gestão operacional (Brad; Brad; 2015; Osita, Onyebuchi \& Nzekwe, 2014; Middleton, 2003), apesar de que a complexidade de alguns modelos de negócios pode dificultar a consecução de uma análise eficiente devido às questões complexas associadas a estruturas organizacionais ou de negócios (Namugeny; Nimmagadda; Reiners, 2019; Ghazinoory; Abdi, and Azadegan-Mehr, 2011; Helms and Nixon, 2010).

A matriz SWOT é composta por quatro combinações de suas dimensões que são chamadas de maxi-maxi (forças / oportunidades), maxi-mini (forças / ameaças), mini-maxi (fraquezas / oportunidades) e mini-mini (fraquezas / ameaças), sua utilização apropriada pressupõe não apenas a correta definição dos fatores em cada dimensão, mas o entendimento das interrelações possíveis (Weihrich 1982). Pontos fortes na análise SWOT são as capacidades ou competências internas que são relevantes para a consecução dos objetivos estratégicos; a geração de valor para clientes; e a geração de vantagem competitiva sustentável (Mintzberg; Ahlstrand; Lampel, 1998; Andrew, 1965, Weihrich 1982). As fraquezas, por outro lado, são fatores internos ou restrições que podem impedir ou dificultar o desempenho de uma organização, culminando com a não consecução de objetivos estratégicos e a perda de vantagem competitiva (Eastwood et al, 2016; Burstein; Holsapple, 2008, Weihrich 1982). Tanto os pontos fortes quanto os pontos fracos são elementos intrínsecos à organização e existem em um estado de constante interação (Middleton, 2003; Mintzberg; Ahlstrand; Lampel, 1998, Porter, 1985, Weihrich 1982). Oportunidades na análise SWOT são fatores ou características que podem favorecer ou facilitar o estabelecimento de negócios com vínculos externos às 
organizações. São fatores externos pelos quais as empresas podem explorar suas vantagens ou criar novas vantagens competitivas em termos de custo, diferenciação ou foco (Osita, Onyebuchi \& Nzekwe, 2014; Mintzberg; Ahlstrand; Lampel, 1998; Porter, 1985, Weihrich 1982) As ameaças lidam com fatores negativos externos à empresa, que podem ameaçar suas vantagens competitivas. É preciso notar as ameaças, por serem extrínsecas às organizações, estão na maior parte das vezes, fora de seu controle (Gürel e Tat, 2017; Eastwood et al, 2016; Evans and Wright, 2009; Burstein; Holsapple, 2008; Porter, 1985; Weihrich 1982).

A análise da literatura especializada leva à conclusão de que não existe um modelo definitivo de aperfeiçoamento da Matriz SWOT (Ghazinoory; Abdi; Azadegan-Mehr, 2011; Helms and Nixon; 2010), que o uso de algoritmos e modelos matemáticos pode ser útil, apesar de tornar a ferramenta mais complexa (Chang and Huang; 2006; Kurttila et al. 2000) e que a Matriz SWOT não pode ser usada isoladamente, mas como um instrumento integrado ao planejamento estratégico e suas mais diversas ferramentas (Domeisen; de Sousa, 2006; Ip; Koo, 2004; Hackbarth; Kettinger, 2000; Lee; Ko; 2000). Também se percebe que existe uma lacuna no que tange a clara definição das dimensões - forças, fraquezas, oportunidades e ameaças ainda que haja diversos métodos matemáticos de auxílio ao exercício das definições, há pouca e esparsa discussão teórica acerca das nuances de cada dimensão e de como melhor defini-las para aperfeiçoar a ferramenta (Ghazinoory; Abdi; Azadegan-Mehr, 2011; Helms and Nixon; 2010; Evans and Wright, 2009).

Assim, dado o imenso número de pesquisas já realizadas acerca da Matriz SWOT assim como as lacunas apontadas pelos autores, o presente trabalho pretende focar em questões puramente conceituais de modo a propor melhorias em sua concepção, entendimento e utilização, e não em ferramentas estratégicas ou matemáticas que possam se adaptar à SWOT, nesse aspecto há outros estudos de considerável variedade e relevância (Ver Quadros 2 e 3).

O desafio dos autores é buscar otimizar a matriz SWOT, incrementando uma consistência lógica que diminua as contradições entre os seus elementos e permita uma maior correlação entre eles, sem, contudo, torna-la desnecessariamente complexa ou diminuir sua flexibilidade e adaptabilidade, o que poderia furtá-la de um de seus principais elementos positivos (Ghazinoory; Abdi; Azadegan-Mehr, 2011; Helms and Nixon; 2010).

Portanto, o presente trabalho objetiva propor uma releitura da análise SWOT, apresentando um modelo adequado às demandas/transformações das organizações modernas, focando em uma definição mais robusta e específica de suas categorias.

\section{Referencial Teórico}

Apesar de sua indiscutível popularidade, a origem do termo "SWOT" é incerta, Helms e Nixon (2010) apontam que já há referências acadêmicas e registros corporativos desde a década de 60, especialmente entre os acadêmicos de Harvard ou ao estrategista Igor Ansoff (Haberg apud Helms and Nixon; 2010; Mintzberg; Ahlstrand; Lampel, 1998), tendo crescido sistematicamente como uma ferramenta fundamental para reduzir a quantidade de informações de modo a melhorar a tomada de decisão em situações complexas.

A influência de Andrew (1965) também foi considerável, por levar em consideração em suas análises as influências de conceitos estratégicos encontrados em autores como Chandler e Drucker, reafirmando a importância da clara definição da missão e dos objetivos estratégicos de longo prazo, inclusive o tipo de negócio que a empresa deverá se tornar. Dentro dessa perspectiva, o conceito de SWOT foi introduzido, ou pelo menos formalizado (Lewa; Narikae, 2017; Mintzberg; Ahlstrand; Lampel, 1998; Andrew, 1965).

Porém, foi Weihrich (1982) quem introduziu a matriz SWOT como uma ferramenta para análise de situação e tomada de decisão, o que levou ao aumento do interesse por essa ferramenta nas décadas subsequentes (Gürel e Tat, 2017; Ghazinoory; Abdi; Azadegan-Mehr, 2011).

As vantagens e desvantagens da Análise SWOT já foram extensamente estudadas na literatura especializada, tendo-se 
assim diversos pontos pacíficos (referência). O Quadro 1 traz os principais pontos positivos e negativos da matriz SWOT, ou suas forças e fraquezas, que são mais comumente observados.

Quadro 1: Forças e Fraquezas da Matriz SWOT.

\begin{tabular}{|l|l|}
\hline \multicolumn{1}{|c|}{ Forças } & \multicolumn{1}{|c|}{ Fraquezas } \\
\hline $\begin{array}{l}\text { Revelar as principais forças, fraquezas, ameaças e } \\
\text { oportunidades é condição sine qua non para o } \\
\text { desenvolvimento do planejamento estratégico. }\end{array}$ & $\begin{array}{l}\text { Revelar os principais pontos fortes e fracos, as ameaças e } \\
\text { oportunidades não leva necessariamente à formulação de uma } \\
\text { estratégia eficaz, pois não pressupõe uma ligação lógica com uma } \\
\text { fase de implementação. }\end{array}$ \\
\hline $\begin{array}{l}\text { Junto com o brainstorming a matriz SWOT é uma das } \\
\text { ferramentas estratégicas mais utilizadas nas organizações, } \\
\text { pois é intuitiva e inclusiva. }\end{array}$ & $\begin{array}{l}\text { Sua ampla utilização e aceitação podem estar mais ligadas à sua } \\
\text { natureza intuitiva que aos resultados que produz. }\end{array}$ \\
\hline $\begin{array}{l}\text { Tem o potencial de direcionar a análise estratégica para } \\
\text { competências essenciais, sendo assim um poderoso } \\
\text { norteador estratégico. }\end{array}$ & $\begin{array}{l}\text { Normalmente faltam informações-chave na análise SWOT } \\
\text { clássica, como a relevância de cada força, fraqueza, ameaça e } \\
\text { oportunidade no cumprimento da visão e metas pretendidas, bem } \\
\text { como compatibilidade dos elementos. }\end{array}$ \\
\hline $\begin{array}{l}\text { Pode ser utilizada para prever comportamento de } \\
\text { mercado. }\end{array}$ & $\begin{array}{l}\text { É 'ateorética', mais baseada em machismos que em análises } \\
\text { objetivas, o que pode torna o exercício de previsão extremamente } \\
\text { arriscado. }\end{array}$ \\
\hline $\begin{array}{l}\text { Tem sido utilizada com níveis variados de sucesso há } \\
\text { mais de 50 anos. }\end{array}$ & $\begin{array}{l}\text { Algumas empresas têm apenas ideias vagas acerca de suas forças, } \\
\text { fraquezas oportunidades e ameaças. }\end{array}$ \\
\hline $\begin{array}{l}\text { Serve como base conceitual para a integração de outras } \\
\text { ideias, princípios e práticas. }\end{array}$ & $\begin{array}{l}\text { Não há nenhum tipo de alternativa visível para resolução de } \\
\text { conflitos na definição dos fatores da matriz. }\end{array}$ \\
\hline $\begin{array}{l}\text { Pode ser aperfeiçoada por I.A., algoritmos e métodos } \\
\text { matemáticos. }\end{array}$ & $\begin{array}{l}\text { A analise SWOT não provê estratégias para implementação das } \\
\text { inferências baseadas em sua aplicação. }\end{array}$ \\
\hline
\end{tabular}

Fonte: Autores, baseado em Costa et al, (2018); Lewa; Narikae, (2017); Ghazinoory; Abdi, and Azadegan-Mehr, (2011); Helms and Nixon, (2010); Zenobia et al (2009); Morris (2005); Hussey (2002); Houben et al (1999); Pickton and Wright (1998).

Devido à imensa variedade de aplicações da matriz SWOT em diferentes organizações e sua imensa flexibilidade, há um esforço consistente de acadêmicos e gestores em combinar a matriz SWOT com outras ferramentas estratégicas, gerenciais e de tomada de decisão de modo a melhorar os resultados obtidos e dar maior fomento ao planejamento estratégico (Ghazinoory; Abdi; Azadegan-Mehr, 2011; Helms and Nixon; 2010). O Quadro 2 traz um resumo de algumas da principais ferramentas que já foram combinadas com a matriz SWOT na ordem que apareceram na literatura especializada, seja para otimizar os resultados obtidos com a matriz original ou para melhorar os resultados da ferramenta em questão, assim como as referências na literatura especializada. 
Quadro 2: Uso combinado da análise SWOT com outras ferramentas gerenciais.

\begin{tabular}{|c|c|}
\hline Ferramenta Estratégica/Gerencial & Autores \\
\hline $\begin{array}{l}\text { Análise de Importância-Desempenho (IPA) - para identificar SWOT com base em } \\
\text { pesquisas de satisfação do cliente, que produz SWOT priorizado correspondente à } \\
\text { percepção dos clientes. }\end{array}$ & Phadermrod; Crowder; Wills, (2019) \\
\hline $\begin{array}{l}\text { TRIZ "teoria da resolução inventiva de problemas" - para obter uma melhor } \\
\text { visualização de elementos 'ocultos' na análise SWOT e para quantificar as } \\
\text { implicações de cada elemento SWOT na resolução de problemas. }\end{array}$ & Brad; Brad (2015); King (2004) \\
\hline $\begin{array}{l}\text { Balance Scorecard - para combinar as quarto perspectivas do BSC com as quatro } \\
\text { dimensões da SWOT de modo a facilitar a discussão sobre oportunidades e/ou } \\
\text { ameaças estratégicas. }\end{array}$ & $\begin{array}{l}\text { Domeisen; de Sousa (2006); Ip and Koo } \\
\text { (2004); Hackbarth; Kettinger (2000); } \\
\text { Lee; Ko (2000). }\end{array}$ \\
\hline $\begin{array}{l}\text { Análise PESTEL - para prover as informações necessárias aos elementos } \\
\text { ambientais da matriz SWOT. }\end{array}$ & $\begin{array}{l}\text { Costa et al (2018); Panagiotou and } \\
\text { Wijnen (2005). }\end{array}$ \\
\hline $\begin{array}{l}\text { Current Reality Tree - Para prover informações necessárias à definição dos fatores } \\
\text { e resolução de conflitos. }\end{array}$ & Coman; Ronen (2009). \\
\hline $\begin{array}{l}\text { Cinco Forças de Porter - para melhor definição do ambiente competitivo e estudo } \\
\text { comparativo com concorrentes. }\end{array}$ & $\begin{array}{l}\text { Costa et al (2018); Evans; Wright } \\
\text { (2009); Hackbarth; Kettinger (2000); } \\
\text { Ruocco; Proctor (1994). }\end{array}$ \\
\hline $\begin{array}{l}\text { Gap analysis - para otimizar a comparação do desempenho real com o desempenho } \\
\text { potencial ou desejado, estabelecendo assim melhores noções sobre forças e } \\
\text { fraquezas. }\end{array}$ & Lin et al (2008). \\
\hline $\begin{array}{l}\text { 3CG - Para entender como o ambiente interno e externo da organização } \\
\text { fetar sua estratégia de portfólio. }\end{array}$ & $\begin{array}{l}\text { Costa et al (2018); McGrail and Roberts } \\
(2005) \text {. }\end{array}$ \\
\hline $\begin{array}{l}\text { Matriz (QFD) - Quality Function Deployment - para levar em consideração as } \\
\text { requisições de projetos ou clientes na definição de forças e fraquezas assim como } \\
\text { dar suporte à decisão estratégica. }\end{array}$ & $\begin{array}{l}\text { Sharma; Rawani, (2008); Ho (2008); Ip; } \\
\text { Koo (2004). }\end{array}$ \\
\hline $\begin{array}{l}\text { Product Life Cycle Analysis - para melhor entender as implicações das } \\
\text { oportunidades e ameaças do ambiente no ciclo de vida dos produtos e serviços, } \\
\text { assim como para mensurar os impactos causados por forças e fraquezas percebidas. }\end{array}$ & $\begin{array}{l}\text { Hricová (2016); Mayer; Vambery } \\
\text { (2008). }\end{array}$ \\
\hline $\begin{array}{l}\text { Total Productive Maintenance Frameworks - para consolidar uma lista de fatores } \\
\text { críticos de sucesso para TPM, além de identificar os pontos fracos e ameaças } \\
\text { potenciais. }\end{array}$ & t al (2008). \\
\hline $\begin{array}{l}\text { Force Field Analysis - para melhor implementação de programas de resistência à } \\
\text { mudança. }\end{array}$ & Oliver; Donelly (2007). \\
\hline $\begin{array}{l}\text { Estratégias de desenvolvimento de Clusters - para priorizar os diferentes clusters } \\
\text { baseados em seu potencial. }\end{array}$ & 2007). \\
\hline $\begin{array}{l}\text { a tornar as conclusões da SWOT mais robustas para a tomada } \\
\text { ão estratégica. }\end{array}$ & $\begin{array}{l}\text { Hossain; Hossain (2015); Lu et al } \\
\text { (2007). }\end{array}$ \\
\hline $\begin{array}{l}\text { mparativa em relação à SWOT, resultando } \\
\text { ada de decisão. }\end{array}$ & Nickols; Ledgerwod (2006). \\
\hline $\begin{array}{l}\text { A) - para lidar com visões divergentes sobre a } \\
\text { ente fraquezas. }\end{array}$ & Ishir \\
\hline $\begin{array}{l}\text { Telescopic observations framework - para aprimorar a coleta de inteligência e } \\
\text { melhorar o planejamento de marketing. }\end{array}$ & $\begin{array}{l}\text { Panagiotou; Van } \\
\text { Panagiotou (2003). }\end{array}$ \\
\hline $\begin{array}{l}\text { Visão baseada em recursos - para melhor analisar os recursos e competências } \\
\text { distintivos das organizações. }\end{array}$ & $\begin{array}{l}\text { Marti (2004); Fahy and Smithee (1999); } \\
\text { Dyson (2004). }\end{array}$ \\
\hline $\begin{array}{l}\text { Análise da Cadeia de Valor - para preencher algumas das lacunas de análise interna } \\
\text { da SWOT deixa como também analisar o ambiente externo além das necessidades e } \\
\text { desejos dos clientes. }\end{array}$ & Grandy; Mills (2004). \\
\hline Modelos de TQM - para dar suporte a implementação da gestão da qualidade total. & $2002)$ \\
\hline $\begin{array}{l}\text { Métodos de análise financeira - para prover insights financeiros nas quatro } \\
\text { dimensões da análise SWOT. }\end{array}$ & los; Chyssochoidis (2000). \\
\hline $\begin{array}{l}\text { Modelo } 7 \mathrm{~S} \text { de McKinsey - para examinar uma organização e identificar os } \\
\text { elementos que podem não estar contribuindo efetivamente para o seu sucesso, } \\
\text { tornando a análise SWOT mais robusta. }\end{array}$ & Kettinger (2000). \\
\hline $\begin{array}{l}\text { Análise de Impacto Cruzado TOWS - para estabelecer priorizações no processo de } \\
\text { decisão estratégica baseado na análise cruzada entre fatores internos e externos. }\end{array}$ & Proctor (2002). \\
\hline $\begin{array}{l}\text { Matriz Espacial - para expandir as análises internas (força financeira e } \\
\text { competitiva) e externas (força da indústria e estabilidade do ambiente) de }\end{array}$ & $\mathrm{R}$ \\
\hline
\end{tabular}


classificar a postura estratégica da organização como: agressiva, competitiva, conservadora ou defensiva.

Matriz do Tempo - para abordar o problema da dimensão do tempo na matriz

SWOT e a complexidade das interações de fatores situacionais.

Fonte: Autores.

Para melhorar a eficácia da matriz SWOT, estabelecendo KPIs assim como instrumentos de quantificação das informações e resultados, muitos pesquisadores a integraram não apenas com outras ferramentas gerenciais como exposto no Quadro 2, mas também com métodos analíticos e quantitativos, dando assim, muitas vezes, um peso numérico a elementos essencialmente subjetivos (Gürel e Tat, 2017; Ghazinoory; Abdi; Azadegan-Mehr, 2011; Ho 2008). No Quadro 3 encontram-se referências com relação às ferramentas quantitativas utilizadas para otimizar a Matriz SWOT.

Quadro 3: Ferramentas Quantitativas de Apoio à Matriz SWOT.

\begin{tabular}{|l|l|}
\hline \multicolumn{1}{|c|}{ Ferramenta Quantitativa } & \multicolumn{1}{|c|}{ Autores } \\
\hline $\begin{array}{l}\text { Medidas - Estabelecimento de critérios de peso para os fatores, a fim de examinar os principais } \\
\text { pontos fortes e fracos em relação a cada questão-chave. }\end{array}$ & Ramos et al. (2000) \\
\hline $\begin{array}{l}\text { Sistemas Baseados em Conhecimento (knowledge-based System - KBS) para auxiliar gerentes } \\
\text { com os elementos mais complexos da análise SWOT. }\end{array}$ & Houben et al. (1999) \\
\hline $\begin{array}{l}\text { Analytic Hierarchy Process (AHP) - para integrar as equipes em decidir aspectos relativos à } \\
\text { matriz SWOT como a classificação dos fatores. }\end{array}$ & $\begin{array}{l}\text { Kurttila et al. } \\
\text { Chang; Huang (2006). }\end{array}$ \\
\hline \begin{tabular}{l} 
Conjunto difuso 'fuzzy sets' - para avaliação, priorização e extração de estratégias. \\
\multicolumn{1}{|c|}{ Kjolstad } \\
Método da Sublista contígua de soma máxima - para criação de algoritmos de apoio à decisão.
\end{tabular} & Lu (2010) \\
\hline $\begin{array}{l}\text { Programação matemática; Meta-heurísticas e Análise de Desenvolvimento de Dados - para dar } \\
\text { robustez às decisões assim como fazer análises mais amplas do ambiente interno externo. }\end{array}$ & Ho (2008) \\
\hline $\begin{array}{l}\text { Análise multicritério de suporte à decisão - para incluir diversas variáveis em decisões } \\
\text { relacionadas à definição dos fatores. }\end{array}$ & Kangas et al (2003) \\
\hline
\end{tabular}

Fonte: Autores.

Percebe-se que através da análise das ferramentas de apoio, um dos grandes desafios por trás da utilização eficiente da Matriz SWOT é a possibilidade de quantificação dos fatores para dar suporte à priorização de elementos-chave no processo de decisão e, em muitos casos, as combinações entre Matriz SWOT e ferramentas matemáticas e estatísticas resultaram em novos modelos de matriz SWOT, que apesar de aparentemente mais robustos, também eram mais complexos e menos intuitivos (Gürel e Tat, 2017; Ghazinoory; Abdi; Azadegan-Mehr, 2011; Lu, 2010; Chang and Huang; 2006).

\section{Metodologia}

Para atingir os objetivos propostos, os autores incorreram em uma pesquisa qualitativa, pois o tema é focado em um nível de realidade que não pode ser, a priori, quantificado, buscando apreender perspectivas, insights e experiências pessoais dos indivíduos e organizações objetos do estudo (Pereira, 2018; Saunders, Lewis \& Thornhill, 2016).

A pesquisa foi dividida em duas etapas: i) pesquisa bibliográfica e documental; e ii) pesquisa-Ação com observação participante (Saunders, Lewis \& Thornhill, 2016; Denscombe, 2010; Argyris, 2004; Yin, 2001).

O primeiro estágio da pesquisa leva em consideração a análise dos trabalhos acadêmicos relativos à Matriz SWOT, de modo a compreender sua evolução histórica, seguido da análise dos documentos da empresa em que o estudo foi realizado (Costa et al, 2018) de modo a acompanhar a evolução do entendimento e da utilização da Matriz SWOT naquela organização.

O segundo estágio da pesquisa integra elementos de pesquisa ação e observação participante, já que se dá em uma organização em que um dos autores ocupou cargo decisório em uma posição de gerência sênior por oito anos na área de vendas 
e desenvolvimento de negócios, o que permitiu implementar estratégias e ter a experiência direta de seu impacto no modus operandi do negócio e em seus resultados. As observações para o atual estudo se deram entre os anos de 2014-2016 (Costa et al, 2018).

A pesquisa ação e de observação participante se deu em uma empresa britânica especializada em business intelligence, conferências e feiras comerciais. Durante o período do estudo, a empresa possuía 25 funcionários divididos principalmente em quatro departamentos: Vendas e Desenvolvimento de Negócios, Marketing, Pesquisa e Desenvolvimento (Produtores de Conferência) e Gerenciamento e Operações de Eventos. Os chefes de cada departamento - exceto Gestão de Eventos e Operações - participam do planejamento estratégico ao lado do Diretor Geral, do Fundador e CEO, bem como do Conselho de Investidores. A empresa atendia a três setores principais, organizando eventos e feiras comerciais internacionais para as indústrias de tecnologias de gestão de ativos financeiros, tecnologias de gestão de recursos humanos e plataformas de jogos de celular, apresentando um faturamento anual de pouco mais de $£ 3,5$ milhões de libras. No período que englobou a pesquisa, a empresa optou por adotar uma estratégia de geoclonagem de seus produtos, ou seja, expandir seus eventos de maior sucesso para outras regiões, mantendo o modelo de negócio, (Costa, 2012) para apoiar seu plano de crescimento e se manter competitiva diante de um número cada vez maior de concorrentes menores, bem como de grandes eventos globais (Costa et al, 2018).

A observação participante teve um papel fundamental para o desenvolvimento do trabalho, tanto seu processo de coleta e análise de dados quanto a análise de resultados, pois o estudo lida com acontecimentos em tempo real, contextualizados dentro da realidade da organização e de sua estratégia competitiva, incorporando elementos mais sutis que envolvem a definição de objetivos, assim como o entendimento de forças e fraquezas. Dada a aproximação do autor com a organização e o interesse da mesma no estudo do tema, não se percebeu reflexibilidade, ou seja, os fatos, acontecimentos e impressões descritas não ocorreram de forma singular ou diferenciada devido à observação dos atores dentro da organização (Costa et al, 2018; Saunders, Lewis \& Thornhill, 2016; Denscombe, 2010; Yin, 2001).

A coleta de dados se deu através de sessões de brainstorming com a diretoria da empresa e seus stakeholders, comparando impressões, objetivos, perspectivas, de modo a dar o máximo de liberdade aos participantes, além da análise dos documentos estratégicos da empresa.

\section{Resultados e Discussão}

Baseados nos levantamentos documentais e empíricos, com vistas à superação dos problemas de precisão na definição das diferentes dimensões da Matriz SWOT (Lewa; Narikae, 2017; Pickton; Wright 1998; Weihrich, 1982), os autores propuseram uma metodologia em que cada categoria da análise SWOT é dividida em cinco subcategorias, oferecendo assim, um guia para dar suporte a análises mais detalhadas e robustas.

É imprescindível notar que determinadas forças, fraquezas, oportunidades e ameaças podem possuir natureza complexa e multifacetada, se encaixando em categorias diferentes, por isso é importante o debate dentro da organização para se definir a categoria mais correspondente, de modo a melhor guiar o planejamento estratégico e a tomada de decisão.

Essas categorias foram criadas na medida em que a diretoria da empresa revia seu planejamento estratégico, em discussões e sessões quadrimestrais de brainstorming entre os anos de 2014 e 2016, fazendo um esforço consciente para integrar a experiência empírica aos insights levantados pela pesquisa bibliográfica para aprimorar as definições das categorias em suas diversas nuances, de modo a criar uma Matriz SWOT mais próxima à realidade. Para cada categoria apresentada abaixo foi apontada uma experiência da própria empresa que levou à sua conceptualização, de modo a fazer com que o exercício de definição de categorias estivesse fundamentado não apenas em bases teóricas, mas também empíricas.

As forças e Fraquezas são divididas nas seguintes categorias: i) sintomáticas (indícios de fenômenos mais profundos); 
ii) Adormecidas (importantes, mas não urgentes); iii) Constantes (de longo prazo); iv) Circunstanciais (repentinas e passageiras); e v) Tangíveis (forças ou fraquezas que estão sendo devidamente exploradas ou remediadas). Os detalhes de cada uma das categorias estão explicitados nos Quadros 4 e 5.

Quadro 4: Tipos de Forças.

\begin{tabular}{|c|l|l|}
\hline Categoria & \multicolumn{1}{|c|}{ Características } & \multicolumn{1}{|c|}{ Exemplo na empresa } \\
\hline Sintomáticas & $\begin{array}{l}\text { São forças aparentes que podem ser sintomas de um } \\
\text { problema ou fraqueza. } \\
\text { Não representam vantagem competitiva de longo } \\
\text { prazo. }\end{array}$ & $\begin{array}{l}\text { Na empresa, notou-se a presença de forças } \\
\text { sintomáticas nos produtos que encontravam } \\
\text { em grau avançado de maturidade. Eles geravam } \\
\text { considerável impacto na receita da empresa, mas } \\
\text { não se podia prever seu ciclo de vida ou estendê- } \\
\text { lo. }\end{array}$ \\
\hline \multirow{5}{*}{ Adormecidas } & $\begin{array}{l}\text { As competências que não são utilizadas na } \\
\text { organização. } \\
\text { São recursos que não possuem resultado ou impacto } \\
\text { no desempenho. } \\
\text { Toda força adormecida é uma candidata a virar } \\
\text { fraqueza. }\end{array}$ & $\begin{array}{l}\text { um time multicultural, essa diversidade não era } \\
\text { utilizada no desenvolvimento de novos produtos, } \\
\text { resultando no desperdício de talentos. }\end{array}$ \\
\hline \multirow{5}{*}{ Constantes } & $\begin{array}{l}\text { São vantagens competitivas sustentáveis, um } \\
\text { diferencial de longo prazo e marca registrada da } \\
\text { organização. } \\
\text { Devem ser preservadas como diferencial } \\
\text { competitivo. }\end{array}$ & $\begin{array}{l}\text { O Brand Value é uma das forças constantes que } \\
\text { foram apontadas na empresa. }\end{array}$ \\
\hline Tangíveis & $\begin{array}{l}\text { Surgem repentinamente a partir mudanças bruscas } \\
\text { no ambiente interno e externo e podem também } \\
\text { sumir sem que se faça nada a respeito. } \\
\text { São vantagens acidentais que se não utilizadas, } \\
\text { podem refletir fraquezas fundamentais. }\end{array}$ & $\begin{array}{l}\text { A capacidade de aumentar a produção ou criar } \\
\text { novos produtos para responder às demandas foi } \\
\text { percebido na empresa como uma força } \\
\text { circunstancial. }\end{array}$ \\
\hline $\begin{array}{l}\text { São as forças que estão sendo utilizadas e cujos } \\
\text { resultados podem ser medidos objetivamente. } \\
\text { Fazem parte da estratégia da empresa, tanto sua } \\
\text { elaboração quanto execução. } \\
\text { Podem ser entendidas como o diferencial } \\
\text { competitivo da empresa. }\end{array}$ & $\begin{array}{l}\text { A tradição de alguns eventos da empresa, apesar } \\
\text { de estarem no nível de 'vaca leiteira' em seu } \\
\text { portfólio (Costa, et al, 2018; Henderson, 2013; } \\
\text { Armstrong; Brodie, 2004), continuava sendo } \\
\text { percebido como uma vantagem tangível, pelo } \\
\text { menos no curto e médio prazo. }\end{array}$ \\
\hline
\end{tabular}

Fonte: Autores.

A distinção destes cinco níveis de força permitiu que as decisões da organização não fossem enviesadas devido às forças sintomáticas (apenas aparente, porém insustentáveis), dando espaço para estratégias focadas nas forças constantes, assim como no esforço de utilizar eficientemente dentro do plano de ação o maior número possível de forças observadas. 
Quadros 5: Tipos de Fraquezas.

\begin{tabular}{|c|c|c|}
\hline Categoria & Características & Exemplo na empresa \\
\hline Sintomáticas & $\begin{array}{l}\text { As fraquezas aparentes que sinalizam fraquezas } \\
\text { mais profundas. } \\
\text { Elas não são o problema, mas um reflexo dele. } \\
\text { Se o gestor não está fazendo nada para suplantar } \\
\text { essa fraqueza, ela é de fato sintomática e é preciso } \\
\text { se fazer algo a respeito. }\end{array}$ & $\begin{array}{l}\text { Um exemplo de fraqueza sintomática percebido } \\
\text { na empresa eram os altos índices de turnover que } \\
\text { era gerado pelo fato de os funcionários utilizarem } \\
\text { a empresa como um trampolim para ganhar } \\
\text { experiência e ir para empresas maiores, o que na } \\
\text { verdade era um sintoma da falta de uma política } \\
\text { de endomarketing. }\end{array}$ \\
\hline Adormecidas & $\begin{array}{l}\text { Podem gerar problemas futuros, mas não são } \\
\text { urgentes. } \\
\text { Geralmente são características de empresas } \\
\text { reativas. }\end{array}$ & $\begin{array}{l}\text { O fato da empresa não utilizar informações } \\
\text { sistemáticas e organizadas sobre seus clientes } \\
\text { para campanhas de marketing mais } \\
\text { individualizadas foi percebido como fraqueza } \\
\text { adormecida. }\end{array}$ \\
\hline Constantes & 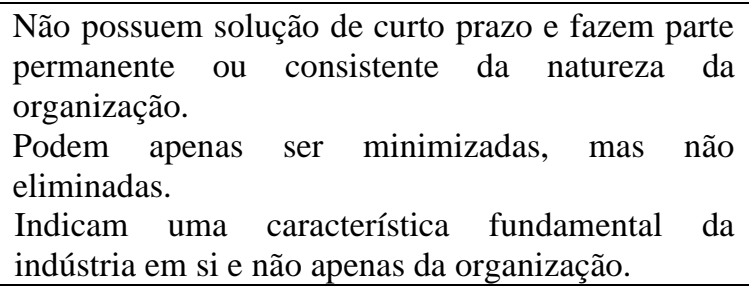 & $\begin{array}{l}\text { O fato de a empresa estudada ser uma pequena } \\
\text { empresa competindo com grandes concorrentes, } \\
\text { foi visto como uma fraqueza constante. }\end{array}$ \\
\hline Circunstanciais & $\begin{array}{l}\text { Surgem repentinamente devido às mudanças } \\
\text { bruscas no ambiente e podem também sumir sem } \\
\text { que se faça nada a respeito. } \\
\text { Porém, indica-se que se trabalhe para superá-las, } \\
\text { pois se deixadas ao acaso podem se agravar. }\end{array}$ & $\begin{array}{l}\text { O fato de a empresa estudada se concentrar em } \\
\text { um pequeno número de indústrias é uma } \\
\text { fraqueza circunstancial que poderia surgir } \\
\text { durante cenários de crise, como a crise de } 2008 \\
\text { que atingiu a indústria de ativos financeiros, a } \\
\text { principal indústria alvo desta empresa (Costa et } \\
\text { al, 2018). }\end{array}$ \\
\hline Tangíveis & $\begin{array}{l}\text { Fraquezas reconhecidas e que estão sendo } \\
\text { remediadas ou possuem uma estratégia de } \\
\text { remediação. } \\
\text { Possuem ampla possibilidade de serem revertidas, } \\
\text { remediadas ou, pelo menos, minimizadas. }\end{array}$ & $\begin{array}{l}\text { Percebeu-se que a ausência da utilização de } \\
\text { tecnologia de big data para as estratégias de } \\
\text { marketing e desenvolvimento de produtos era } \\
\text { uma franqueza tangível, pois estava sendo } \\
\text { ativamente endereçada pela gestão (Costa et al, } \\
\text { 2018). }\end{array}$ \\
\hline
\end{tabular}

Fonte: Autores.

A distinção destes cinco níveis de fraqueza foi fundamental, especialmente o reconhecimento das fraquezas sintomáticas - sintomas de uma fraqueza maior não reconhecida - assim foi possível analisar mais profundamente as causas das fraquezas percebidas na organização. $O$ entendimento sobre as fraquezas circunstanciais também permitiu modelos de planejamento e ação estratégica mais flexível, levando em conta esses potenciais problemas.

As oportunidades foram divididas nas seguintes categorias: i) Exploradas; ii) Passageiras; iii) Desperdiçadas; iv) Inacessíveis; e v) Disfarçadas (podem ser, na verdade, ameaças). Percebeu-se que as ameaças se encaixavam em categorias ontologicamente distintas. Os detalhes desta categoria estão explicitados no Quadro 6. 
Quadro 6: Tipos de Oportunidades.

\begin{tabular}{|c|c|c|}
\hline Categoria & Características & Exemplo na empresa \\
\hline Exploradas & $\begin{array}{l}\text { A empresa aproveita ou possui planejamento de } \\
\text { curto prazo para aproveitar essas oportunidades. } \\
\text { Representa o próprio foco de atuação da empresa e a } \\
\text { pedra de toque de toda sua estratégia. }\end{array}$ & $\begin{array}{l}\text { No estudo, notou-se a presença de oportunidades } \\
\text { exploradas, especificamente na adaptação das } \\
\text { agendas dos eventos às novas tecnologias como } \\
\text { Big Data (Costa et al, 2018). }\end{array}$ \\
\hline Passageiras & $\begin{array}{l}\text { Sazonais e insustentáveis, essas oportunidades são } \\
\text { vagas e requerem explorações de baixo custo. } \\
\text { Exigem adaptabilidade, pois possuem prazo de } \\
\text { validade e poucas barreiras de entrada. }\end{array}$ & $\begin{array}{l}\text { A empresa norteava sua estratégia competitiva à } \\
\text { clonagem geográfica dos eventos (Costa, 2012); } \\
\text { todavia, temas paralelos que surgiam eram } \\
\text { incorporados em eventos feitos para explorar a } \\
\text { demanda do mercado em curto prazo apenas. }\end{array}$ \\
\hline Desperdiçadas & $\begin{array}{l}\text { Apesar de estarem ao alcance da empresa, essas } \\
\text { oportunidades não estimulam a movimentação } \\
\text { estratégica - podem ser sintomas de fraquezas } \\
\text { intrínsecas. } \\
\text { Dão abertura para concorrência de novos } \\
\text { produtos/serviços ou novos modelos de negócios. }\end{array}$ & $\begin{array}{l}\text { A empresa notou oportunidades desperdiçadas } \\
\text { para criação de conteúdo e inteligência de } \\
\text { mercado - especialmente webinars quando ainda } \\
\text { não eram amplamente populares; todavia, a falta } \\
\text { de pessoal especializado na área impediu que } \\
\text { fossem aproveitadas. }\end{array}$ \\
\hline Inacessíveis & $\begin{array}{l}\text { Não apresentam uma boa relação de custo vs. } \\
\text { benefício e a empresa decide não explorar. } \\
\text { Porém, podem ser sintomas de fraquezas } \\
\text { intrínsecas - como a incapacidade de adaptar-se a } \\
\text { novas demandas. } \\
\text { Geralmente são exploradas pelos líderes de } \\
\text { mercado apenas. }\end{array}$ & $\begin{array}{l}\text { O fato de a empresa possuir pouca liquidez } \\
\text { devido ao tamanho aponta diversas } \\
\text { oportunidades inacessíveis em termos de } \\
\text { expansão para mercados onde havia demanda } \\
\text { para seus produtos/serviços, mas o custo era } \\
\text { simplesmente proibitivo. }\end{array}$ \\
\hline Disfarçadas & $\begin{array}{l}\text { São oportunidades que podem facilmente virar } \\
\text { ameaças. } \\
\text { Estão inseridas em uma complexa configuração de } \\
\text { fatores externos e internos. } \\
\text { Geralmente a ausência de barreiras para entrada de } \\
\text { competidores se configura nesse tipo de } \\
\text { oportunidade. }\end{array}$ & $\begin{array}{l}\text { O fato dos patrocinadores dos eventos da } \\
\text { empresa estarem dispostos a dar suporte à } \\
\text { estratégia de geoclonagem, apesar de ser uma } \\
\text { oportunidade, poderia esconder a ameaça de que } \\
\text { os mercados escolhidos ainda não possuíam } \\
\text { maturidade para receber os eventos. }\end{array}$ \\
\hline
\end{tabular}

Fonte: Autores.

A diferenciação das oportunidades permitiu à empresa distinguir mais eficientemente as decisões estratégicas que deveriam ser tomadas. Especialmente o entendimento das oportunidades disfarçadas permitiu à empresa se antecipar a futuros problemas.

Por fim, as ameaças apresentam as seguintes categorias: i) Constantes (não podem ser neutralizadas); ii) Passageiras; iii) Adormecidas; iv) Provocadas; e v) Disfarçadas (podem ser, de fato, oportunidades). Os detalhes desta categoria se encontram no Quadro 7:

Quadro 7: Tipos de Ameaças.

\begin{tabular}{|l|l|l|}
\hline \multicolumn{1}{|c|}{ Categoria } & \multicolumn{1}{|c|}{ Características } & \multicolumn{1}{|c|}{ Exemplo na empresa } \\
\hline Constantes & $\begin{array}{l}\text { Não podem ser neutralizadas, apenas remediadas, } \\
\text { quando muito. } \\
\text { Toda decisão estratégica precisa levar em } \\
\text { consideração essas ameaças, pois elas representam } \\
\text { riscos de sobrevivência. } \\
\text { Podem ser sintoma de problemas internos de } \\
\text { adaptação. }\end{array}$ & $\begin{array}{l}\text { As } \\
\text { estratégia de geoclonagem era uma ameaça } \\
\text { constante ao sucesso de eventos, especialmente } \\
\text { na Ásia. }\end{array}$ \\
\hline Passageiras & $\begin{array}{l}\text { São temporárias e só representam ameaças dentro } \\
\text { de um quadro especifico de fraqueza das empresas. } \\
\text { São como infecções oportunistas que se aproveitam } \\
\text { das fraquezas das empresas. }\end{array}$ & $\begin{array}{l}\text { As recessões, por exemplo, foram vistas como } \\
\text { ameaças passageiras, não obstante perigosas, já } \\
\text { que a empresa não possuía liquidez. }\end{array}$ \\
\hline Adormecidas & $\begin{array}{l}\text { A conjuntura de fatores que pode gerar essa ameaça } \\
\text { não se forma, mas está constantemente presente. }\end{array}$ & $\begin{array}{l}\text { A tentativa de copiar os eventos da empresa } \\
\text { pelos concorrentes maiores eram ameaças }\end{array}$ \\
\hline
\end{tabular}




\begin{tabular}{|c|c|c|}
\hline & $\begin{array}{l}\text { Devem ser levadas em consideração em todo } \\
\text { planejamento de logo prazo e exigem uma } \\
\text { abordagem contingencial das organizações. }\end{array}$ & $\begin{array}{l}\text { adormecidas, que poderiam surgir caso houvesse } \\
\text { um aumento súbito do mercado. }\end{array}$ \\
\hline Provocadas & $\begin{array}{l}\text { Ameaças genéricas ou adormecidas que são atraídas } \\
\text { para a organização devido à sua estratégia ou falta } \\
\text { dela. } \\
\text { Uma vez despertas, podem apresentar um poder } \\
\text { destrutivo avassalador. }\end{array}$ & $\begin{array}{l}\text { O investimento em mercados não maduros, } \\
\text { baseado na demanda dos patrocinadores dos } \\
\text { eventos poderia gerar desgaste de imagem e } \\
\text { perda de ganhos futuros, caso o evento } \\
\text { fracassasse em número de atendentes. }\end{array}$ \\
\hline Disfarçadas & $\begin{array}{l}\text { Podem se tornar oportunidades caso a empresa se } \\
\text { adapte. } \\
\text { Sua natureza vai depender diretamente das forças e } \\
\text { fraquezas da organização. }\end{array}$ & $\begin{array}{l}\text { A ascensão do LinkedIn, que foi considerada } \\
\text { inicialmente como uma ameaça para a indústria } \\
\text { de eventos pois permitia formas alternativas de } \\
\text { networking na verdade foi uma grande } \\
\text { oportunidade para a empresa coletar dados e } \\
\text { melhorar seu marketing. }\end{array}$ \\
\hline
\end{tabular}

Fonte: Autores.

O entendimento das diferentes categorias de ameaças foi vital, pois deu à empresa maiores perspectivas quanto à priorização de decisões. Um esforço consciente foi feito para avaliar a possibilidade das ameaças serem, de fato, oportunidades disfarçadas, dando assim novos rumos às decisões estratégicas.

\section{Conclusão}

O presente trabalho buscou demonstrar como a utilização da Análise SWOT em uma empresa britânica de inteligência corporativa entre os anos de 2014 e 2016 evoluiu através da introdução de novas categorias de forças, fraquezas, oportunidades e ameaças.

A pesquisa foi, em parte, motivada pela constatação de que a aplicação da Matriz SWOT poderia estar incorrendo no erro de ser apenas um exercício mal estruturado e generalista, refletindo mais a percepção dos participantes do que propriamente a natureza dos fatos. De modo a superar esse problema, buscou-se estudar a ferramenta a fundo, de modo a desenvolver níveis aceitáveis de conhecimento técnico e conceitual entre os participantes, assim como encorajar a comunicação aberta no exercício de definição dos fatores e na análise dos desdobramentos gerados pelos planejamentos estratégicos da empresa.

Como resultado a empresa otimizou a utilização da ferramenta, podendo assim melhor discernir suas forças e fraquezas como também avaliar oportunidades e ameaças, dando um suporte mais robusto ao processo de tomada de decisões e levando a mudanças estruturais em seus produtos (agenda de eventos) e processos (mais tempo alocado para atividades de P\&D). A empresa, para construir vantagem competitiva sustentada, alinhando as suas forças com o mercado externo, utilizava além da Matriz SWOT, diversas técnicas e ferramentas tradicionais de planeamento estratégico (Análise PESTE, as 5 Forças de Porter e Matriz BCG), a utilização dessas ferramentas também foi melhorado devido às melhorias implementadas na análise SWOT (Costa et al, 2018).

Este trabalho contribui com a literatura e a prática da gestão estratégica ao oferecer uma alternativa para endereçar as dificuldades de precisão na definição das dimensões da Matriz SWOT, criando subcategorias até então não levantadas na literatura para que se possa mais precisamente classificar forças e fraquezas e avaliar oportunidades e ameaças, renovando este instrumento de planejamento estratégico.

As limitações desse trabalho se encontram em duas categorias distintas: i) limitações de ordem prática - discernir categorias é um exercício que pode levar a uma melhor estruturação do planejamento estratégico e da tomada de decisão, todavia, os problemas relativos ao subjetivismo da análise permanecem válidos, mesmo que mitigados; ii) o universo da pesquisa - é preciso notar que essas categorias foram definidas a partir da experiência de profissionais em uma empresa 
específica, essa experiência ainda que válida como ponto de partida para análises mais profundas, não pode ser tomada como um modelo definitivo para releitura da Matriz SWOT.

Como sugestão para futuros trabalhos os autores veem dois caminhos inter-relacionados: i) realizar pesquisas similares em outras organizações para buscar apreender a existência de outras possíveis subcategorias das dimensões da Matriz SWOT; ii) integrar essa nova proposta de modelo com ferramentas de análise mais robustas, de modo a poder quantificar e medir o peso das diferentes categorias no planejamento e ação estratégica da empresa. Alguns estudos em conjuntos difusos (Emblemsvag, Kjolstad, 2002) e análise multicritério (Kangas et al, 2003) podem nortear esse estágio de evolução da pesquisa.

\section{Referências}

Andrews, K. R. (1965) The Concept of Corporate Strategy. Dow Jones-Irwin.

Argyris, C. (1994). Knowledge for Action. Jossey-Bass.

Brad, S., \& Brad, E. (2015). Enhancing SWOT analysis with TRIZ-based tools to integrate systematic innovation in early task design. Procedia Engineering. 131 (2015) 616 - 625. https://doi.org/10.1016/j.proeng.2015.12.455

Chong, J. K. s. (2004). Six steps to better crisis management. Journal of Business Strategy. 25(2):43-46. https://doi.org/10.1108/02756660410525407

Burstein, F.; \& Holsapple, C. W. (eds) (2008) Handbook on Decision Support Systems 1: Basic Themes. Springer Science \& Business Media.

Chang, H. H., \& Huang, W. C. (2006). Application of a quantification SWOT analytical method. Mathematical and Computer Modelling. https://doi.org/10.1016/j.mcm.2005.08.016

Coman, A., \& Ronen, B. (2009). Focused SWOT: Diagnosing critical strengths and weaknesses. International Journal of Production Research. 47 (20), 5677-5689. https://doi.org/10.1080/00207540802146130

Costa, J. F Jr., Rezende, J. F. D. de, Cabral, E. L. dos S., Florentino, D. R. de M., \& Soares, A. R. (2018). The Impact of Big Data on SME`s Strategic Management: A Study on a Small British Enterprise Specialized in Business Intelligence. Journal of Management and Strategy. 9(4), 10-31 https://doi.org/10.5430/jms.v9n4p10

Costa, J. F. Jr. (2012) The Marketing Concept: How Osneymedia ltd is attempting to adopt a culture of marketing orientation. London School of Commerce Cardiff Metropolitan University.

Denscombe, M. (2010). The Good Research Guide: For small-scale social research projects. Open University Press.

Domeisen, N., \& de Sousa, P. (2006). SWOT analysis: NGOs as partners. International Trade Forum.

Dyson, R. G. (2004). Strategic development and SWOT analysis at the University of Warwick. European Journal of Operational Research. 152 (2004) 631640. https://doi.org/10.1016/S0377-2217(03)00062-6

Culp III, K., Eastwood, C., Turner, S., Goodman, M., \& Ricketts, K. G. (2016). Using a SWOT analysis: Taking a look at your organization. Community and Economic Development Publications. 3.

Egan, T. M., \& Lancaster, C. M. (2005). Comparing Appreciative Inquiry to Action Research: Organization Development Journal. 23(2), 29-49. 21p

Emblemsvåg, J., \& Endre Kjølstad, L. (2002). Strategic risk analysis - a field version. Management Decision. 40(9):842-852 https://doi.org/10.1108/00251740210441063

Evans, C.; \& Wright, A. (2009). How to conduct a SWOT analysis. The British Journal of Administrative Management, $24,10-34$.

Fahy, J. \& Smithee, A. (1999). Strategic marketing and the resource based view of the firm. Academy of Marketing Science Review, 19(10).

Ghazinoory, S., Abdi, M., \& Azadegan-Mehr, M. (2011). Swot methodology: A state-of-the-art review for the past, a framework for the future. Journal of Business Economics and Management. 12(1):24-48 10.3846/16111699.2011.555358

Grandy, G., \& Mills, A. J. (2004). Strategy as simulacra? A radical reflexive look at the discipline and practice of strategy. Journal of Management Studies. 41, 7: 1153-1170 10.1111/j.1467-6486.2004.00470.x

Grover, S., Agrawal, V. P., \& Khan, I. A. (2005). Human resource performance index in TQM environment. International Journal of Management Practice. 1 (2), 131 - 151. 10.1504/IJMP.2005.007132

Gürel, M., \& TAT, M. (2017) SWOT analysis: A Theoretical Review, The Journal of International Social Research, 10(51):994-1006. $10.17719 /$ jisr.2017.1832

Hackbarth, G., \& Kettinger, W. J. (2000). Building an e-business strategy. Information Systems Management. 17 (3) 78-93 10.1201/1078/43192.17.3.20000601/31243.10 
Helms, M. M., \& Nixon, J. (2010). Exploring SWOT analysis - where are we now? Journal of Strategy and Management. 3 (3) 215-251 $10.1108 / 17554251011064837$

Hricová, B., Lumnitzer, E., Piňosová, M., \& Goga Bodnárová, A. (2016). Life-cycle assessment of product through of the SWOT analysis. Production Management and Engineering Sciences - Scientific Publication of the International Conference on Engineering Science and Production Management, ESPM 2015. 10.1201/b19259-18

Ho, W. (2008). Integrated analytic hierarchy process and its applications - A literature review. European Journal of Operational Research. 186, (1), 211-228 10.1016/j.ejor.2007.01.004

Houben, G., Lenie, K., \& Vanhoof, K. (1999). Knowledge-based SWOT-analysis system as an instrument for strategic planning in small and medium sized enterprises. Decision Support Systems. 26 (2) 125-135 10.1016/S0167-9236(99)00024-X

Hussey, D. (2002). Company analysis: determining strategic capability. Strategic Change. 11: 43-52. 10.1002/jsc.568

Ip, Y. K., \& Koo, L. C. (2004). BSQ strategic formulation framework. Managerial Auditing Journal. 19(4):533-543. 10.1108/02686900410530538

Kangas, J., Kurttila, M., Kajanus, M., \& Kangas, A. (2003). Evaluating the management strategies of a forestland estate - The S-O-S approach. Journal of Environmental Management. 69 (4) 349-358. 10.1016/j.jenvman.2003.09.010

Kasturi, S. P., \& Gransberg, D. D. (2002). Time management-a design-build builder’s perspective. Cost Engineering (Morgantown, West Virginia).

King, R. K. (2004). Enhancing SWOT analysis using triz and the bipolar conflict graph: a case study on the Microsoft Corporation. Proceedings of TRIZCON2004, 6th Annual Altshuller Institute.

Koufopoulos, D. N., \& Chryssochoidis, G. M. (2000). The effects of an uncertain country environment upon leadership and strategic planning practices. Strategic Change. 9, 379-395. 10.1002/1099-1697

Kurttila, M., Pesonen, M., Kangas, J., \& Kajanus, M. (2000). Utilizing the analytic hierarchy process (AHP) in SWOT analysis - A hybrid method and its application to a forest-certification case. Forest Policy and Economics. 1 (1) 41-52. 10.1016/s1389-9341(99)00004-0

Lee, S. F., \& Sai on ko, A. (2000). Building balanced scorecard with SWOT analysis, and implementing "Sun Tzu's The Art of Business Management Strategies" on QFD methodology. Managerial Auditing. 15(1/2), 68-76 Journal. 10.1108/02686900010304669

Lewa, P. M., \& Narikae, P. O. (2017). The Origins and Development of Strategic Management "Knowledge": a Historical Perspective. European Journal of Business and Strategic Management. 2(6), 1-19.

Lin, C., Hsu, M. L., Liu, A., \& Ju-Chuan, J. C. (2008). Pursuing excellence in firm core knowledge through intelligent group decision support system. Industrial Management \& Data Systems. 108 (3) 277-296. 10.1108/02635570810858723

Lu, K. H.; Lin, C. T.; \& Huang, I. C. (2007) Editorial: some measurement methods are applied to business performance management. International Journal of Business Performance Management, 9(1)

Lu, W. (2010). Improved SWOT Approach for Conducting Strategic Planning in the Construction Industry. Journal of Construction Engineering and Management. 10.1061/(asce)co.1943-7862.0000240

Marti, J. (2004). 'Strategic knowledge benchmarking system (SKBS): A knowledge-based strategic management information system for firms'. Journal of Knowledge Management, 8, 6, 31-49

Martin, A. (2002). The globalisation of Welsh business through the development of managerial competencies and behavioural attitudes in e-business to ebusiness. Managerial Auditing Journal. 17 (6) 333-337. 10.1108/02686900210434122

Mayer, P., \& Vambery, R. G. (2008). Aligning Global Business Strategy Planning Models With Accelerating Change. Journal of Global Business \& Technology. 4, 31-48.

McGrail, M., \& Roberts, B. (2005). Strategies in the broadband cable TV industry: The challenges for management and technology innovation. 7 (1) 53-65. doi: $10.1108 / 14636690510578270$

Middleton, J. (2003) The ultimate Strategy Library. Capstone Publishing.

Mintzberg, H., Ahlstrand, B., \& Lampel, J. (1998). Strategy Safari: A Guide through the Wilds of Strategic Management. Free Press.

Mishra, R. P., Anand, G., \& Kodali, R. (2008). A SWOT analysis of total productive maintenance frameworks. International Journal of Management Practice. 3(1), 51-81 10.1504/IJMP.2008.016047

Namugenyi, C., Nimmagadda, S. L., \& Reiners, T. (2019). Design of a SWOT analysis model and its evaluation in diverse digital business ecosystem contexts. Procedia Computer Science. 159 (2019) 1145-1154. 10.1016/j.procs.2019.09.283

Nickols, F., \& Ledgerwood, R. (2006). The Goals Grid as a tool for strategic planning. Consulting to Management. 17(1), 36-38.

Novicevic, M. M., Harvey, M., Autry, C. W., \& Bond, E. U. (2004). Dual-perspective SWOT: A synthesis of marketing intelligence and planning. Marketing Intelligence \& Planning. 22(1), 84-94. 10.1108/02634500410516931

Oliver, G. R., \& Donnelly, P. J. (2007). Effective use of a Strategic Issue Management System (SIMS): combining tools and approach. Journal of Public Affairs. 2007; 7:399-406.10.1002/pa.278 
Research, Society and Development, v. 10, n. 2, e25710212580, 2021

(CC BY 4.0) | ISSN 2525-3409 | DOI: http://dx.doi.org/10.33448/rsd-v10i2.12580

Osita, I., Onyebuchi, I., \& Nzekwe, J. (2014). Organization's stability and productivity: the role of SWOT analysis an acronym for strength, weakness, opportunities and threat. International Journal of Innovative and Applied Research. 2 (9)

Panagiotou, G. (2003). Bringing SWOT into focus. In Business Strategy Review. 14 (2). 10.1111/1467-8616.00253

Panagiotou, G., \& van Wijnen, R. (2005). The "telescopic observations" framework: An attainable strategic tool. In Marketing Intelligence and Planning. 23(2):155-171. 10.1108/02634500510589912

Phadermrod, B., Crowder, R. M., \& Wills, G. B. (2019). Importance-Performance Analysis based SWOT analysis. International Journal of Information Management. 1-10 https://doi.org/10.1016/j.ijinfomgt.2016.03.009

Pereira A. S. et al. (2018). Metodologia da pesquisa científica. UFSM.

Pickton, D. W., \& Wright, S. (1998). What's swot in strategic analysis? Strategic Change. 7, 101-109. 10.1002/(sici)1099-1697(199803/04)7:2<101::aidjsc332>3.0.co;2-6

Porter, M. E. (1985). The Competitive Advantage: Creating and Sustaining Superior Performance. Free Press.

Proctor, R. A. (1992). Structured and Creative Approaches to Strategy Formulation. In Management Research News. 15(1), 13-18. 10.1108/eb028190

Reid, N., Carroll, M. C., \& Smith, B. W. (2007). Critical Steps in the Cluster Building Process. Economic Development. 6 (4), 44.

Ruocco, P., \& Proctor, T. (1994). Strategic Planning in Praetiee: A Creative Approaeh. Marketing Intelligence \& Planning. 10.1108/02634509410069056

Saunders, M.; Lewis, P.; \& Thornhill, A. (2016) Research Methods for Business Students, (7th edition). Essex: Pearson Education.

Sharma, J. R; \& Rawani, A. M. (2008). Quality Function Deployment: Integrating Comprehensive Matrix and SWOT Analysis for Effective Decision Making. Journal of Industrial Engineering International. 4(6), 19-31

Weihrich, H. (1982). The TOWS matrix-A tool for situational analysis. Long Range Planning. 15 (2) 54-66 https://doi.org/10.1016/0024-6301(82)90120-0

Yin, R. (2001). Estudo de caso: planejamento e método. Bookman.

Zenobia, B., Weber, C., \& Daim, T. (2009). Artificial markets: A review and assessment of a new venue for innovation research. Technovation. 29(5):338-350 https://doi.org/10.1016/j.technovation.2008.09.002) 